\title{
Immune MAL2-practice: breast cancer immunoevasion via MHC class I degradation
}

\author{
Devin Dersh and Jonathan W. Yewdell \\ Laboratory of Viral Diseases, National Institute of Allergy and Infectious Diseases, NIH, Bethesda, Maryland, USA.
}

\begin{abstract}
The success of tumor immunotherapy, while partial, confirms the existence and importance of tumor immunosurveillance. $\mathrm{CD8}^{+} \mathrm{T}$ cell recognition of tumor-specific peptides bound to MHC class I (MHC-I) molecules is central to this process. In this issue of the $J C I$, Fang, Wang, et al. describe a unique tumor immunoevasion strategy based on endocytosis and degradation of MHC-I complexes mediated by the trafficking factor MAL2. Notably, MAL2 expression was associated with poor prognosis of breast cancer, and its downregulation enhanced CD8 ${ }^{+} \mathrm{T}$ cell recognition of breast cancer in various experimental models. This work demonstrates that a deeper understanding of tumor interference with MHC-I stability and trafficking has considerable potential for enhancing immunotherapies.
\end{abstract}

\section{Tumor immunosurveillance and evasion}

Tumor immunoevasion is a multifaceted process encompassing expression of immune checkpoints, cytokine production in the tumor microenvironment, and impaired innate and adaptive immune detection of malignant cells. The recent explosion of successful immunotherapies places antigen processing and presentation center stage in T cell-mediated immunosurveillance. Nearly all nucleated cells express MHC class I (MHC-I). By presenting small peptides derived from endogenous proteins, MHC-I enables CD ${ }^{+} \mathrm{T}$ cells to surveil foreign and abnormal translation products, including viral proteins and tumor-associated antigens.

The principal elements of the MHC-I antigen processing pathway have been established in the past 25 years (Figure 1). The proteasome degrades both immature and mature proteins to generate cytosolic peptides (1). The transporter associated with antigen processing (TAP) imports proteasome-generated cytosolic pep- tides into the endoplasmic reticulum (ER) where peptides assemble with MHC-I with the assistance of dedicated and general purpose molecular chaperones, followed by trafficking to the cell surface via the standard secretory pathway.

The effectiveness of MHC-I-based immunosurveillance is evident from the ubiquitous evolution of viral proteins that specifically interfere with antigen processing $(1,2)$. Similarly, many tumors impair antigen processing and peptide presentation to avoid immune eradication $(3,4)$, a process termed immunoevasion or immunoediting (5). How do tumors sabotage this system? We have likely only scratched the surface of this question, which is critical for distinguishing patients that are most likely to respond to immunotherapy and for devising treatments to convert resistant tumors. The standard approach has predominantly focused on sequencing tumor nucleic acids. This is no surprise, as a needle biopsy provides ample material for determining the transcriptome, exome, or whole genome. Tumor immunoevasion studies have focused mainly on

Related Article: https://doi.org/10.1172/JCI140837

Conflict of interest: The authors have declared that no conflict of interest exists.

alterations in the canonical components of the class I assembly complex (6), largely ignoring the fate of correctly generated MHC-I peptide complexes. Yet tumors can also exploit MHC-I trafficking and degradation to evade the immune response.

\section{MAL2 misdirects MHC-I}

Enter Fang, Wang, et al., who in this issue of the JCI identified genes from publicly available breast cancer data sets that correlate with poor patient survival (7). One top hit, MAL2, was unusual; it failed to regulate breast cancer growth either in vitro or in immunocompromised mice. However, in normal mice, MAL2 expression correlated with tumor size, suggesting a critical role when tumors are under immune pressure. Sure enough, in human triple-negative breast cancers (cancers lacking receptors for estrogen, progesterone, and epidermal growth factor), MAL2 expression in bulk transcriptome and single-cell RNA sequencing data sets correlated with low $\mathrm{CD}^{+} \mathrm{T}$ cell cytotoxic signatures, suggesting that MAL2 expression suppresses MHC-I-based immunosurveillance (7).

Fang, Wang, et al. used cell models that expressed peptides from ovalbumin or the human cancer-testis antigen NY-ESO-1 to show that tumor MAL2 inhibits $\mathrm{T}$ cell responses during $\mathrm{T}$ cell coculture. MHC-I-blocking antibodies abolished the effects of MAL2 expression, indicating that the inhibitory function of MAL2 could be directly attributed to antigen presentation. Thus, a battery of diverse T cellbased assays provide evidence that MAL2 impairs breast cancer antigen presentation, crippling the $\mathrm{T}$ cell response (7).

MAL2 is a 176 residue multispanning transmembrane protein known to participate in transcytosis $(8,9)$. Pursuing the unique link between antigen presentation and MAL2, Fang, Wang, et al. found that MAL2 abundance indeed controlled cell surface levels of MHC-I. Mass spectrometry analysis of epitope-tagged MAL2-associated proteins identified both MHC-I and 


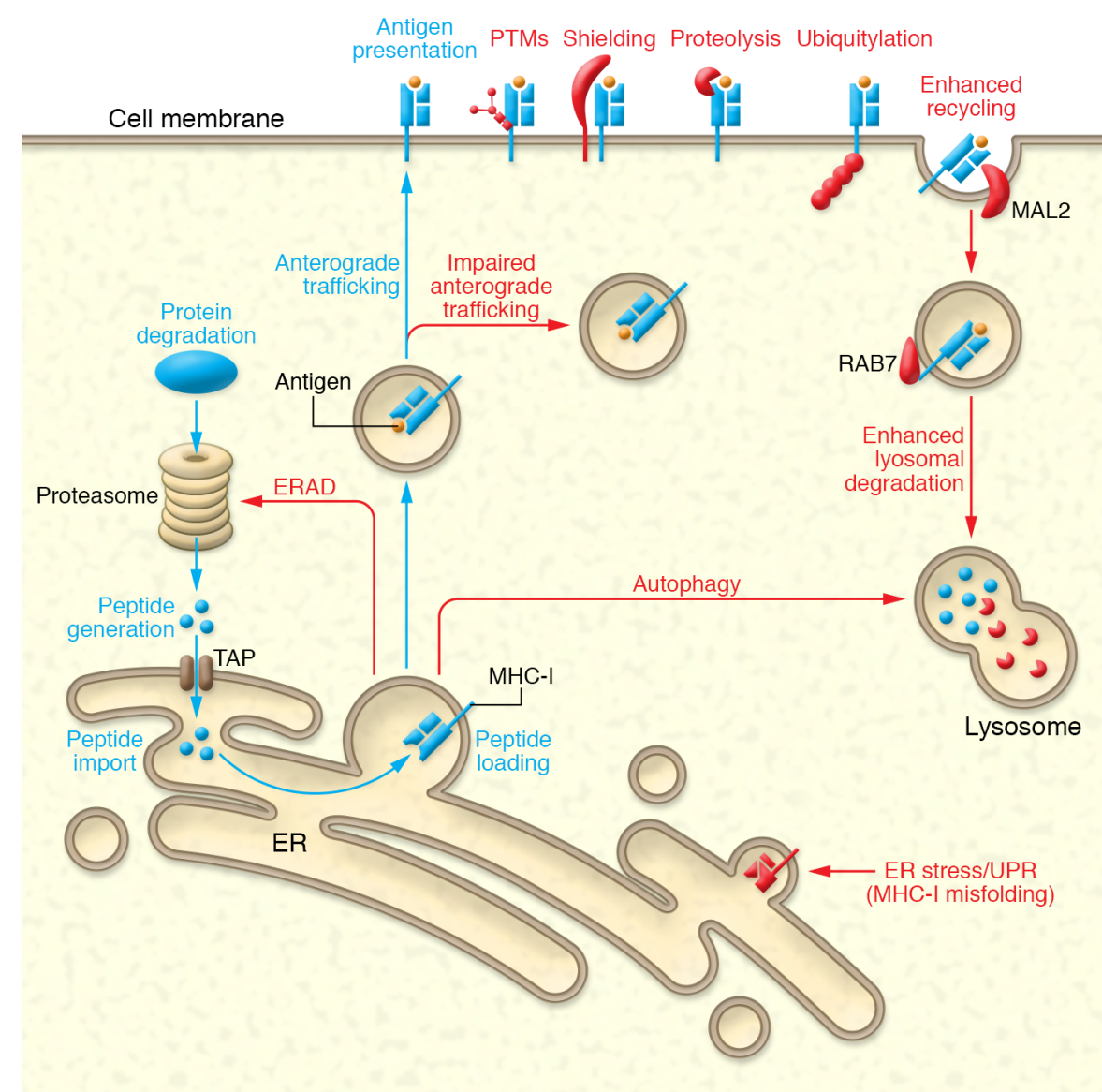

RAB7, an endosome recycling factor (10). After confirming the RAB7-MHC-I interaction by coimmunoprecipitation, Fang, Wang, et al. showed that MAL2 overexpression enhanced the MHC-I-RAB7 interaction, including on cell-derived endosomes (7). Notably, MAL2 overexpression enhanced MHC-I degradation, which was blocked by filipin, an endocytosis inhibitor (7). These findings are consistent with MAL2-RAB7-mediated endosomal/lysosomal degradation of MHC-I.

Fang, Wang, and colleagues next examined MAL2 function in vivo using a mouse breast cancer model to generate single-cell RNA sequencing data for tens of thousands of tumor-infiltrating $\mathrm{CD}^{+}$ $\mathrm{T}$ cells. The sequencing data matched their analyses of published human data sets, showing that tumor MAL2 expression impaired the T cell cytotoxicity signature and furthermore strongly reduced the number of infiltrating effector $\mathrm{T}$ cells (7). Extending these experiments, the authors showed that MAL2 overexpression blocked autologous $\mathrm{T}$ cells from targeting primary human breast cancer organoids.
Finally, immunohistochemical staining of hundreds of primary human biopsies for MAL2, CD8, and cytolytic granules (GZMB) demonstrated that tumors with high MAL2 protein levels displayed relatively weak $\mathrm{CD}^{+} \mathrm{T}$ cell activity (7).

Altogether, Fang, Wang, et al. provide robust evidence for an inhibitory effect of MAL2 on T cell recruitment and cytotoxicity in breast cancers, based on posttranslational downregulation of MHC-I (7).

\section{Clinical implications}

Immunoevasion is a critical step in the development of clinical tumors. Malignant cells frequently hijack existing cell biological processes, e.g., coopting epigenetic regulation of MHC-I machinery (11) to reduce levels of antigen processing and presentation components. Subversion of MHC-I trafficking by MAL2 fits this modus operandi by utilizing preexisting protein trafficking pathways. It will be of great interest to determine the extent to which other tumors exploit MAL2 misdirection of MHC. Consistently, MAL2 is overexpressed in colorectal cancer (12),

Figure 1. Model for the MHC-I antigen processing pathway and evasion strategies. Oligopeptides destined for MHC-I complexes are typically generated in the cytosol by proteasome-mediated degradation. Peptides are imported into the endoplasmic reticulum (ER) by TAP, the transporter associated with antigen processing. The ER lumen is the site of heterotrimer assembly, where heavy chains, $\beta 2$-microglobulin, and a high-affinity peptide associate while bound to TAP with the assistance of dedicated and general purpose chaperones. Assembled MHC-I is delivered to the cell surface via the Golgi complex. Cancer cells often interfere in this process to evade CD8 ${ }^{+} \mathrm{T}$ cells, although current understanding of immunoevasion has focused on genetic, transcriptomic, and signaling alterations. Highlighted in red are putative and known pathways that can contribute to immunoevasion at the posttranslational level. Fang, Wang, et al. (7) showed that MAL2 promotes endocytosis to reduce tumor antigens on the surface of breast cancer cells. ERAD, ER-associated degradation; PTMs, posttranslation modifications; UPR, unfolded protein response.

renal cell carcinoma (13), and ovarian carcinoma (14). It is important to note that NK-based immunosurveillance of MHC-I-deficient cells constrains tumor escape and necessitates that tumor cells deploy additional changes to walk the razor's edge between $\mathrm{NK}$ and $\mathrm{CD} 8^{+} \mathrm{T}$ cell immune pressure.

The findings described by Fang, Wang, et al. (7) raise the unexplored possibility of a MAL2-based therapy, either in the form of inhibitors or even anti-MAL2 monoclonal antibodies, given that MAL2 was predominantly expressed on the surface of breast cancer cells. This MAL2 therapy could be particularly important in combination with antibodies targeting PD-L1/ PD-1 checkpoints.

Although posttranslational manipulation of antigen presentation has been historically less studied than genetic and transcriptomic analyses, the work of Fang, Wang, et al. serves as a shining example of how trafficking and protein-centric mechanisms can be relevant to immunebased control over tumors. Appropriately, recently published studies expand on 
these modes of regulation in a number of cancers: (a) In triple-negative breast cancer, expression of the long noncoding RNA LINK-A enhances ubiquitin-mediated degradation of peptide loading complex components, hampering MHC-I folding and peptide loading (15). (b) In pancreatic ductal adenocarcinoma, MHC-I is strongly downregulated via autophagy and the cargo receptor NBR1 (16). Inhibition of autophagy restored MHC-I surface levels and improved checkpoint blockade therapies in mouse models. (c) In diffuse large B cell lymphoma, unbiased genome-wide CRISPR screens identified dozens of previously unknown antigen presentation regulators, spanning epigenetics, transcription, peptide loading, and protein trafficking (17). Numerous proteins implicated in endocytosis and endosomal/lysosomal delivery were identified as negative regulators, likely delineating lymphoma-specific recycling and degradation pathways. (d) Desialylating cell surface MHC-I heavy chain is associated with decreased degradation (18), which may have important implications for (frequently hypersialylated) cancer cells. Oncogenes themselves may even play direct roles in MHC-I internalization and immune escape (19).

The cancer-specific examples of regulation discussed above highlight some of the myriad mechanisms that tumor cells exploit to evade T cells - additional putative avenues for posttranslational control of antigen presentation are depicted in Figure 1. Clearly, there are avenues galore for aspiring cancer cell biologists to explore tumor antigen processing and ultimately generate new drugs and treatments to enhance cancer immunotherapy.

\section{Acknowledgments}

DD and JWY are supported by the NIH Intramural Research Program.

Address correspondence to: Jonathan W. Yewdell, Room 2e13C.1, Bldg 33, NIH, Bethesda, Maryland 20892, USA. Phone: 301.402.4502; Email: jyewdell@nih.gov.

1. Yewdell JW, Hill AB. Viral interference with antigen presentation. Nat Immunol. 2002;3(11):1019-1025.

2. Schuren $A B$, et al. Recent advances in viral evasion of the MHC class I processing pathway. Curr Opin Immunol. 2016;40:43-50.

3. Restifo NP, et al. Identification of human cancers deficient in antigen processing. J Exp Med. 1993;177(2):265-272.

4. Elliott BE, et al. Perspectives on the role of MHC antigens in normal and malignant cell development. Adv Cancer Res. 1989;53:181-245.

5. Dunn GP, et al. Cancer immunoediting: from immunosurveillance to tumor escape. Nat Immunol. 2002;3(11):991-998.

6. Dersh D, et al. A few good peptides: MHC class I-based cancer immunosurveillance and immunoevasion [published online August 20, 2020]. Nat Rev Immunol. https://doi.org/10.1038/ s41577-020-0390-6.

7. Fang Y, et al. MAL2 drives immune evasion in breast cancer by suppressing tumor antigen presentation. J Clin Invest. 2021;131(1):e140837.

8. de Marco MC, et al. MAL2, a novel raft protein of the MAL family, is an essential component of the machinery for transcytosis in hepatoma HepG2 cells. J Cell Biol. 2002;159(1):37-44.

9. de Marco MC, et al. Dynamics of MAL2 during glycosylphosphatidylinositol-anchored protein transcytotic transport to the apical surface of hepatoma HepG2 cells. Traffic. 2006;7(1):61-73.

10. van der Kant R, et al. Cholesterol-binding molecules MLN64 and ORP1L mark distinct late endosomes with transporters ABCA3 and NPC1. JLipid Res. 2013;54(8):2153-2165.

11. Burr ML, et al. An evolutionarily conserved function of polycomb silences the MHC class I antigen presentation pathway and enables immune evasion in cancer. Cancer Cell. 2019;36(4):385-401.

12. Li J, et al. The four-transmembrane protein MAL2 and tumor protein D52 (TPD52) are highly expressed in colorectal cancer and correlated with poor prognosis. PLoS One. 2017;12(5):e0178515.

13. Rohan S, et al. Gene expression profiling separates chromophobe renal cell carcinoma from oncocytoma and identifies vesicular transport and cell junction proteins as differentially expressed genes. Clin Cancer Res. 2006;12(23):6937-6945.

14. Byrne JA, et al. MAL2 and tumor protein D52 (TPD52) are frequently overexpressed in ovarian carcinoma, but differentially associated with histological subtype and patient outcome. BMC Cancer. 2010;10:497.

15. Hu Q, et al. Oncogenic IncRNA downregulates cancer cell antigen presentation and intrinsic tumor suppression. Nat Immunol. 2019;20(7):835-851.

16. Yamamoto K, et al. Autophagy promotes immune evasion of pancreatic cancer by degrading MHC-I. Nature. 2020;581(7806):100-105.

17. Dersh D, et al. Genome-wide screens identify lineage- and tumor-specific genes modulating MHCI- and MHC-II-restricted immunosurveillance of human lymphomas [published online December 2, 2020]. Immunity. https://doi.org/10.1016/ j.immuni.2020.11.002.

18. Silva Z, et al. MHC class I stability is modulated by cell surface sialylation in human dendritic cells. Pharmaceutics. 2020;12(3):E249.

19. Bradley SD, et al. BRAF(V600E) co-opts a conserved MHC class I internalization pathway to diminish antigen presentation and $\mathrm{CD}^{+} \mathrm{T}$-cell recognition of melanoma. Cancer Immunol Res. 2015;3(6):602-609. 